\title{
Homology Modelling and Molecular Docking Study of Voltage Gated I on Channels for their R ole in Plant Abiotic Stress
}

\author{
Ravi Ranjan Kr. Niraj, Nikita Singh, Sween and Ajit Kumar* \\ Toxicology and Computational Biology Group, Centre for Bioinformatics, M.D. University, Rohtak, Haryana - 124 001, India.
}

\begin{tabular}{|c|c|}
\hline ARTICLE INFO & ABSTRACT \\
\hline $\begin{array}{l}\text { Article history: } \\
\text { Received on: } 13 / 07 / 2014 \\
\text { Revised on: } 28 / 07 / 2014 \\
\text { Accepted on: } 12 / 08 / 2014 \\
\text { Available online: } 27 / 08 / 2014\end{array}$ & $\begin{array}{l}\text { Voltage-gated ion channels (VGICs) are responsible for generation of electrical signals in cell membranes. They } \\
\text { exist mainly in three major forms namely, VGKC (Voltage-gated potassium channel), VGCC (Voltage-gated } \\
\text { calcium channel), and VGSC (Voltage-gated sodium channel). VGICs have been studied extensively in animal } \\
\text { system, especially for their role in electrical signalling during nerve conduction. Their existence in plant system } \\
\text { has been related from very early period of evolution but their role in plant system has not been studied intensively }\end{array}$ \\
\hline $\begin{array}{l}\text { Key words: } \\
\text { Docking, Homology } \\
\text { modelling, Salt stress; } \\
\text { Thermal stress }\end{array}$ & $\begin{array}{l}\text { and is a less explored area. Therefore, the present study was undertaken to investigate the role of VGICs in plant } \\
\text { stress response, abiotic stress in particular, using in-silico tool of docking simulation. No solved crystal structure } \\
\text { of plant VGICs were available at Protein Databank for the purpose of docking studies. Therefore, 3D-structures } \\
\text { of three different VGICs (VGCC, VGKC and VGSC) were constructed using homology modelling tool of } \\
\text { SWISS-MODEL and were selected after structure evaluation. These structures were subjected to docking } \\
\text { simulation against major soil salts and fertilizers. While conducting molecular docking simulation studies, it was } \\
\text { observed that VGICs seems to have negligible role in simple salts physiology like NaCl or KCl, while VGKC } \\
\text { showed good binding pattern with ammonium nitrate and ammonium sulfate, reflecting its significant role in } \\
\text { ammonium -ion physiology. Also, phosphoric acid binding was found significant towards VGKC. } \\
\text { Superphosphate ions and Calcium nitrate showed a good binding pattern towards VGCC while VGSC showed } \\
\text { good affinity for nitrate, phosphate, sodium and ammonium-ions. Also, during simulated annealing docking, it } \\
\text { was observed that binding of phosphoric acid (or phosphate ion) increased at both extreme temperature ends } \\
\text { (lower and higher). The study has provided a good platform for further investigation to establish the role of } \\
\text { VGICs in plant stress response and correlated to other living systems like animals, fungi, etc. }\end{array}$ \\
\hline
\end{tabular}

\section{INTRODUCTION}

Voltage gated ion channel have a crucial role in excitable cells, allowing a rapid and co-ordinated depolarization in response to triggering voltage change [1]. The first voltagedependent ion channel that was isolated and purified was extracted from the eel electroplax where there is a large concentration of $\mathrm{Na}$ channels [2]. Several years later, the sequence of the eel $\mathrm{Na}$ channel was deduced from its mRNA [3]. The first $\mathrm{K}^{+}$channel sequence was deduced from the Shaker mutant of Drosophila melanogaster [4]. These initial sequences were the basis to subsequent cloning of a large number of $\mathrm{Na}, \mathrm{K}$ and $\mathrm{Ca}$ channels in many different species. VGICs are of great importance in plants as they are involved in many activities for instance like voltage gated calcium channel (VGCC) are involved

\footnotetext{
* Corresponding Author

Dr. Ajit Kumar, Assistant Professor, Toxicology and Computational Biology Group, Centre for Bioinformatics M.D. University, Rohtak, Haryana-124001(India).E-mail: akumar.cbt.mdu@gmail.com
}

in maintaining cellular homeostasis, signal transduction etc. Salinisation of agricultural land threatens world food production because it exposes crops to low water potential and high concentration of toxic ions in the soil. In particular, all major crops are sensitive to high concentrations of sodium $\left(\mathrm{Na}^{+}\right)$[5]. Due to the negative electrical potential inside cells $\mathrm{Na}^{+}$influx into plant roots can occur through ion channels or other membrane transport proteins that facilitate passive diffusion of $\mathrm{Na}^{+}$across the plasma membrane.

The combined evidence suggests that cytoplasmic $\mathrm{Na}^{+}$concentrations are generally in the low millimolar range. This is in accordance with the notion that cytoplasmic $\mathrm{Na}^{+}$ concentrations above $100 \mathrm{mM}$ are toxic due to the detrimental effects of a high $\mathrm{Na}^{+}$environment to protein stability [6] and displacement of $\mathrm{K}^{+}$from essential co-factor binding sites on $\mathrm{K}^{+}$-dependent enzymes [7]. Numerous pharmacological and cell biological studies have suggested that voltage-dependent $\mathrm{Ca}^{2+}$ channels in the plasma membrane are important for initiation of plant responses to environmental, hormonal, and pathogenic signals [8]. 
Direct measurements of such channels in plant cells have been reported recently in patch clamp studies [9], radioactive tracer flux studies using plasma membrane vesicles [10,11], and reconstitution studies [12]. These plant $\mathrm{Ca}^{2+}$ channels are activated by membrane depolarization, a characteristic typical of voltagedependentCa ${ }^{2+}$ channels in other systems. Voltage gated potassium channels (VGKC) are involved in regulation of cell volume and the flow of salt across epithelia. Moreover (VGCC) are also involved in opening and closure of stomatal aperture in response to stress conditions [13].

Voltage-gated $\mathrm{Ca}^{2+}$ channel from guard cells are involved in early events of plant hormone-induced responses [14-16]. VGCC from Arabidopsis roots and Daucus carota suspension protoplasts have been shown to be involved in cation uptake, maintaining appropriate electrochemical gradients important for the transport of other ions and cell volume regulation and signalling mechanisms and priming the cell for response [17, 18].

The abiotic stress is considered as the inappropriate condition for the living organism in its environment caused by non-living factors or "environmental factors". In plants, these factors confer an adverse affect on the growth rate and the productivity of the crops. It is, thus important to study the role of abiotic stress in plant system [19]. So the present study was carried out to investigate plant VGICs structures and their functional correlation in abiotic stress in plant system using in-silico tool of molecular docking simulation.

\section{MATERIALS AND METHODS}

\subsection{Collection of protein sequence data of plant VGICs}

The protein sequence of plant VGICs was collected from NCBI [20]. A total of 15 different sequence entries for VGKC were found and retrieved, out of which the sequence of AtKAT1 Arabidopsis thalianagi| 44888080| sp| Q39128.2| KAT1_ARATH was selected for further studies. Similarly 8 different protein sequence for VGCC were retrieved out of which AtTPC1 Arabidopsis thaliana gi|75166464|sp|Q94KI8.1| was selected and 4 different protein sequence for VGSC were found and retrieved out of which VGSC superfamily Micromonaspusilla CCMP1545 gi|303285434|ref|XP_003062007.1| was selected for further studies.

\subsection{Homology modelling}

Homology modelling of the VGIC-proteins was done since no native solved crystal structure of the plant VGICs was available in PDB. The structures of the VGKCs, VGCCs and VGSCs, selected above, were generated using the SWISS MODEL [21] in auto-template mode. The generated models were evaluated using WHAT IF online server [22] for each of the protein models. The different evaluated models, each for VGKCs, VGCCs and VGSCs were selected thus obtained were energy minimised using UCSF-Chimera [23].

\subsection{Docking analysis of VGICs with soil salts and fertilisers}

For the docking analysis, the structures of the ligands namely the salts- $\mathrm{KCl}$ and $\mathrm{NaCl}$ and fertilisers namely- $\left(\mathrm{NH}_{4}\right)_{2} \mathrm{SO}_{4}$, urea, $\mathrm{NH}_{4} \mathrm{NO}_{3}$, single super phosphate, triple super phosphate (TSP), $\mathrm{H}_{3} \mathrm{PO}_{4}, \mathrm{Ca}\left(\mathrm{NO}_{3}\right)_{2}, \mathrm{KNO}_{3}$, mono-ammonium phosphate, diammonium phosphate monocalcium phosphate and $\mathrm{K}_{2} \mathrm{SO}_{4}$ were constructed and energy minimized using the Chemsketch [24] software.

After structure optimization, these structures were converted in .pdb file format using Open Babel [25] software and further energy minimized using UCSF-Chimera. These energy minimized ligands were docked against the modelled protein namely AtKat1 (VGKC of A. thaliana), AtTpc1 (VGCC of $A$. thaliana) and Mpccmp1545 (VGSC of $M$. pusilla) and the interacting residues and the binding energy were noted. For this Autodock 4.2 [26] was used to prepare, run and analyse the docking simulations.

Lamarckian model of genetics, were used in which environmental adaptations of an individual's phenotypes are reverse transcribed into its genotype and become heritable traits. Only polar hydrogen was added to the protein and Kollman and Gastegier charges were assigned. The spacing between grid points was set to default value of $0.375 \AA$. The grid box was set to $480 \times$ $260 \times 280$ (x, y and $\mathrm{z}$ axis) to include all the amino acid residues that were present in protein. A total of 50 independent runs were performed with a step sizes of $0.2 \AA$ for translations and $5^{0}$ for orientations and torsions. The maximum number of generations was set to 1000 and maximum number of top individuals that automatically survived was set to 1 with mutation rate of 0.02 , crossover rate of 0.8 , cluster tolerance $0.5 \AA$, external grid energy 1000.0.

\subsection{Simulated annealing using Autodock was done at different temperatures}

The docking simulation was performed for selected ligands against all the three modelled VGICs, as referred above, using simulated annealing method for analysing the effect of temperature on binding pattern of these proteins for ligands. The selection of ligands was made on the basis of lower binding energies obtained when docked using Lamarckian model of genetics. For this purpose, $\mathrm{NaCl}, \mathrm{H}_{3} \mathrm{PO}_{4}, \mathrm{TSP},\left(\mathrm{NH}_{4}\right)_{2} \mathrm{SO}_{4}$ and $\mathrm{NH}_{4} \mathrm{NO}_{3}$ were selected as ligands while the simulated annealing docking studies were done at 8 different temperatures- $10^{\circ} \mathrm{C}$, $15^{\circ} \mathrm{C}, 25^{\circ} \mathrm{C}, 30^{\circ} \mathrm{C}, 35^{\circ} \mathrm{C}, 40^{\circ} \mathrm{C}, 45^{\circ} \mathrm{C}$ and $50^{\circ} \mathrm{C}$ and the binding energy were noted.

\section{RESULTS AND DISCUSSION}

\subsection{Homology modelling}

The structures of the selected VGICs, namely VGKC (AtKAT1 Arabidopsis thalianagi| 44888080| sp| Q39128.2|), VGCC (AtTPC1 Arabidopsis thaliana gi|75166464|sp|Q94KI8.1|) and VGSC (Micromonaspusilla CCMP1545 gi|303285434 |ref|XP_003062007.1|) were generated using the SWISS MODEL 
in auto-template mode. These models (Figure 1a-c), when evaluated using online WHAT-IF server, showed the Q-mean score, Z-score Ramachandran and Z-score that reflects the acceptability of the models (Table 1). Hence they were used for further docking studies after energy minimization and dockprep using UCSF-Chimera.

\subsection{Docking analysis of VGICs with soil salts and fertilizers}

To investigate the role of plant VGICs in plant stress response, abiotic stress in particular, the modelled VGICs were individually docked against different soil salts and fertilizers. The docking analysis revealed that the binding energy (B.E.) of VGKC ranged from $-0.9 \mathrm{kCal} / \mathrm{mol}$ to $-4.57 \mathrm{kCal} / \mathrm{mol}$ while that of $\mathrm{VGCC}$ and VGSC range from $-0.86 \mathrm{kCal} / \mathrm{mol}$ to $-3.67 \mathrm{kCal} / \mathrm{mol}$ and from $0.83 \mathrm{kCal} / \mathrm{mol}$ to $-4.6 \mathrm{kCal} / \mathrm{mol}$, respectively (Table $2-4$ ). The docking results reflect that VGICs seems to have negligible role in simple salts physiology like $\mathrm{NaCl}$ or $\mathrm{KCl}$, while $\mathrm{VGKC}$ showed good binding pattern with ammonium nitrate and ammonium sulfate, reflecting its significant role in ammonium-ion physiology.
Also, phosphoric acid binding was found significant towards VGKC. Superphosphate ions and Calcium nitrate showed a good binding pattern towards VGCC while VGSC showed good affinity for nitrate, phosphate, sodium and ammonium -ions. Also, arginine was observed to be important in binding of salts to VGKC while aromatic amino acid tyrosine/phenyl alanine was observed to be commonly important for binding of salts to VGCC. Lysine/serine/Leucine was observed to be important in binding of salts to VGSC (Figure 2a-c).

\subsection{Simulated annealing using Autodock was done at different temperatures.}

Simulated annealing was performed for the selected ligands against all the three modelled proteins for the analysis of effect of different temperatures on the binding pattern of the proteins with the ligands at different temperatures. During simulated annealing docking, it was observed that binding of phosphoric acid (or phosphate ion) increased at both extreme temperature ends (lower and higher) as reflected in Table 5-7.

Table. 1: Parametric evaluation of models if VGICs generated by SWISS MODEL.

\begin{tabular}{lllll}
\hline Model name & Template used & Q-mean & Z- score & Ramachandran Plot Z-Score \\
\hline VGKC of A. thaliana (AtKat1) & 4110 & 0.5 & -3.29 & -0.461 \\
VGKC of . thaliana (AtKat1) & $4 \mathrm{f} 41$ & 0.24 & -4.68 & -1.607 \\
VGSC of $M$. pusilla (Mpccmp1545) & 4dck & 0.44 & -3.93 & -0.343 \\
\hline
\end{tabular}

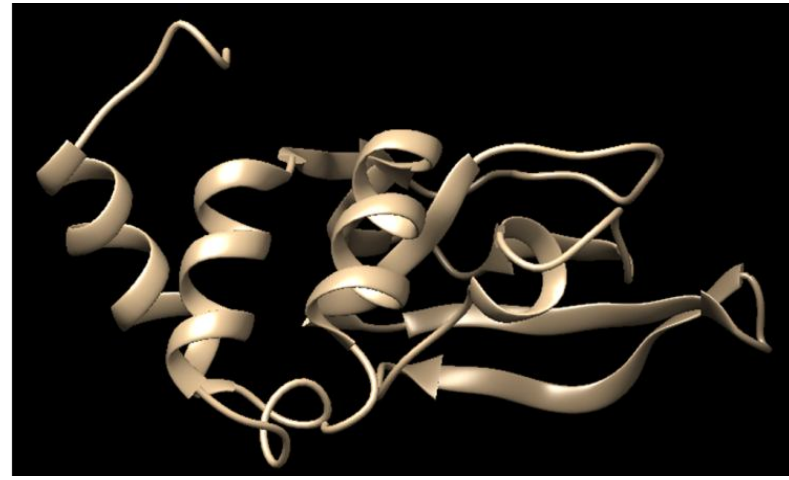

$\mathbf{A}$

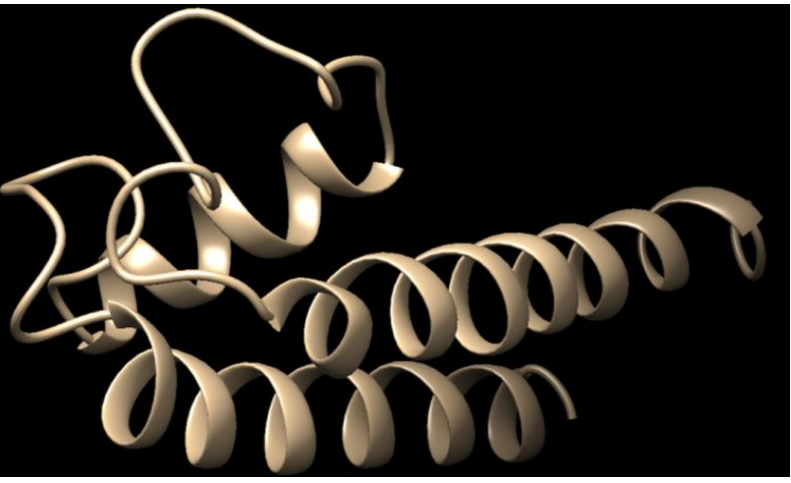

B

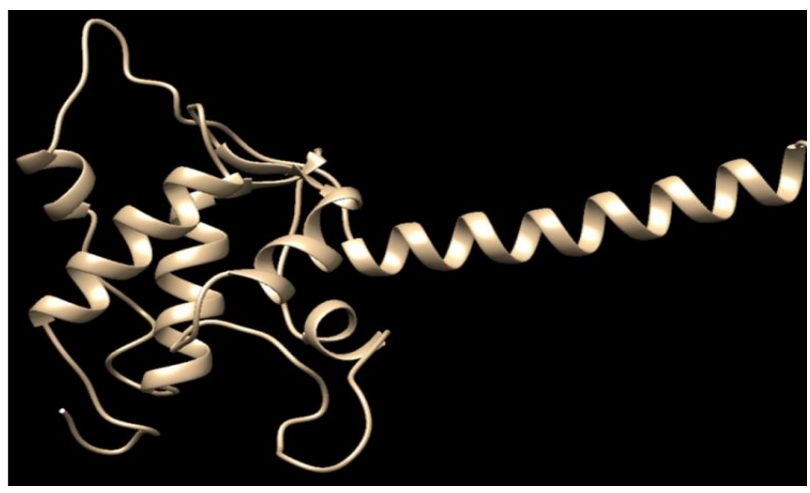

C

Fig. 1: a-c: Homology Models of VGICs of plant systems [a: VGKC of A. thaliana (AtKat1); b: VGCC of A. thaliana (AtTpc1); c: VGSC of M. pusilla (Mpccmp1545)] 
Table. 2: Docking analysis of VGKC of A. thaliana (AtKat1) against different soil salts and fertilizers.

\begin{tabular}{|c|c|c|c|c|c|}
\hline S.No. & Salt name & $\begin{array}{c}\text { B.E. } \\
(\mathrm{kCal} / \mathrm{mol})\end{array}$ & $\mathbf{K i}$ & Interacting residues & H-Bond forming residues \\
\hline 1 & Urea & -3.93 & $1.31 \mathrm{mM}$ & Ile404, Ile405, Glu409, Thr412, Tyr415 & Glu409, Thr412, Tyr415 \\
\hline 2 & $\mathrm{NH}_{4} \mathrm{NO}_{3}$ & -4.39 & $605.53 \mathrm{uM}$ & Lys357, Arg360 & Lys357, Arg360 \\
\hline 3 & SSP & -4.05 & $1.08 \mathrm{mM}$ & Arg307, Arg310, Arg314, Tyr397 & Arg305, Arg310, Arg314 \\
\hline 4 & $\mathrm{H}_{3} \mathrm{PO}_{4}$ & -5.05 & $197.24 \mathrm{uM}$ & Arg305, Lys347, Asp394, Ala393, Glu390 & Lys347, Ala 395 \\
\hline 5 & $\mathrm{NaCl}$ & -0.96 & $198.17 \mathrm{mM}$ & Ile375, Leu377, Thr443, Leu493, Lys496 & - \\
\hline 6 & $\mathrm{KCl}$ & -0.9 & $217.08 \mathrm{mM}$ & Ile375,Leu377,Thr443,Leu493, Lys496 & - \\
\hline 7 & $\mathrm{Ca}\left(\mathrm{NO}_{3}\right)_{2}$ & -4.57 & $445.14 \mathrm{uM}$ & Arg305, Arg307, Arg310, Arg314 & $\operatorname{Arg} 307, \operatorname{Arg} 310, \operatorname{Arg} 314$ \\
\hline 8 & TSP & -4.54 & $4.52 .88 \mathrm{uM}$ & Arg305, Arg307, Arg310, Asp311, Tyr397 & $\operatorname{Arg} 305, \operatorname{Arg} 307$ \\
\hline 9 & $\mathrm{KNO}_{3}$ & -3.72 & $1.88 \mathrm{mM}$ & Gln348, Gln349, Glu350, Asp392, Ser471 & Asp392, Ser471 \\
\hline 10 & $\left(\mathrm{NH}_{4}\right)_{2} \mathrm{SO}_{4}$ & -4.03 & $1.11 \mathrm{mM}$ & Gln344, Glu350, Ser391, Asp392, Asp394 & Gln349, Asp392, Asp394 \\
\hline 11 & $\mathrm{NaNO}_{3}$ & -3.91 & $1.36 \mathrm{mM}$ & $\operatorname{Arg} 305, \operatorname{Arg} 310, \operatorname{Arg} 314$ & $\operatorname{Arg} 310, \operatorname{Arg} 314$ \\
\hline 12 & MAP & -3.21 & $4.44 \mathrm{mM}$ & Arg305, Thr306, Arg307, Arg310, Arg314 & $\operatorname{Arg} 305, \operatorname{Arg} 307, \operatorname{Arg} 310, \operatorname{Arg} 314$ \\
\hline 13 & DAP & -3.6 & $2.28 \mathrm{mM}$ & Arg305, Thr306, Arg307, Arg310, Asp311, Arg314 & $\operatorname{Arg} 305, \operatorname{Arg} 307, \operatorname{Arg} 310, \operatorname{Arg} 314$ \\
\hline 14 & MCP & -3.89 & $1.41 \mathrm{mM}$ & Ile 405 , Ala410, Pro411, Thr412, Tyr415 & Ala410, Thr412, Tyr415 \\
\hline
\end{tabular}

SSP: Single Sugar Phosphate; TSP: Triple Sugar Phosphate; MAP: monoammonium phosphate;

DAP: Diammonium phosphate; MCP: Monocalcium phospate

Table. 3: Docking analysis of VGCC of A. thaliana (AtTpc1) against different soil salts and fertilizers.

\begin{tabular}{llcclc}
\hline S.No. & Salt name & $\begin{array}{c}\text { BE } \\
(\mathbf{k C a l} / \mathbf{m o l})\end{array}$ & $\mathbf{K i}$ & \multicolumn{1}{c}{ Interacting residues } & H-Bond forming residues \\
\hline 1 & $\mathrm{Urea}$ & -3.15 & $4.87 \mathrm{mM}$ & Phe238, Thr241, Gln242, Ser277, Ser278 & Ser278, Glu242 \\
2 & $\mathrm{NH}_{4} \mathrm{NO}_{3}$ & -2.22 & $23.49 \mathrm{mM}$ & Asn267, Phe285, Val289 & Asn267 \\
3 & $\mathrm{SSP}$ & -3.54 & $2.56 \mathrm{mM}$ & Asn267, Pro268, Val270, Ala274, Tyr275 & Val270, Tyr275 \\
4 & $\mathrm{H}_{3} \mathrm{PO}_{4}$ & -3.07 & $5.66 \mathrm{mM}$ & Tyr275, Ser277, Arg279 & Ser277, Arg279 \\
5 & $\mathrm{NaCl}$ & -1.05 & $169.67 \mathrm{mM}$ & Leu227, Ser231, Leu255, Met258 & - \\
6 & $\mathrm{KCl}$ & -0.86 & $233.82 \mathrm{mM}$ & Trp223, Phe226, Ile291, Gly292, Phe295 & Tyr275, Lys276 \\
7 & $\mathrm{Ca}\left(\mathrm{NO}_{3}\right)_{2}$ & -3 & $6.37 \mathrm{mM}$ & Pro244, Pro268, Val270, Pro273, Ala274, Tyr275, Lys276 & Val270, Tyr275 \\
8 & $\mathrm{TSP}$ & -3.67 & $2.05 \mathrm{mM}$ & Pro268, Asp269, Val270, Ile272, Ala274, Tyr275, Ser 281, Ser282 & Asp240 \\
9 & $\mathrm{KNO}_{3}$ & -3.17 & $4.78 \mathrm{mM}$ & Phe238, Glu239, Asp240, Thr241 & Val270, Ile272 \\
10 & $\left(\mathrm{NH}_{4}\right)_{2} \mathrm{SO}_{4}$ & -3.58 & $2.38 \mathrm{mM}$ & Pro268, Asp269, Val270, Ile272, Pro273 & Lys276 \\
11 & $\mathrm{NaNO}_{3}$ & -2.89 & $7.61 \mathrm{mM}$ & Tyr275, Lys276, Arg279 & Tyr216,Tyr305, Asp306 \\
12 & $\mathrm{MAP}$ & -2.69 & $10.59 \mathrm{mM}$ & Thr215, Tyr216, Ala302, Tyr305, Asp306 & Tyr216 \\
13 & $\mathrm{DAP}$ & -3.05 & $5.83 \mathrm{mM}$ & Tyr216 & \\
14 & $\mathrm{MCP}$ & -3.17 & $4.71 \mathrm{mM}$ & Ala410, Thr412, Tyr415 & Pro268, Val270 \\
\hline
\end{tabular}

SSP: Single Sugar Phosphate; TSP: Triple Sugar Phosphate; MAP: monoammonium phosphate;

DAP: Diammonium phosphate; MCP: Monocalcium phospate

Table. 4: Docking analysis of VGSC of A. thaliana (Mpccmp1545) against different soil salts and fertilizers.

\begin{tabular}{|c|c|c|c|c|c|}
\hline S.No. & Salt name & $\begin{array}{c}\text { BE } \\
(\mathrm{kCal} / \mathrm{mol})\end{array}$ & Ki & Interacting residues & H-Bond forming residues \\
\hline 1 & Urea & -3.36 & $3.42 \mathrm{mM}$ & Lys1680, Asp1682, Ser1684, Asp1685 & Lys1680, Asp1682, Ser1684 \\
\hline 2 & $\mathrm{NH}_{4} \mathrm{NO}_{3}$ & -4.6 & $422.13 \mathrm{uM}$ & Leu1627, Lys1628, Asn 1629, Lys 1630 & Lys1628, Lys 1630 \\
\hline 3 & SSP & -3.54 & $2.56 \mathrm{mM}$ & Tyr1595, Phe1639, Gln1640, Arg 1642, Ile1643, His1644, Phe 1687 & Tyr275 \\
\hline 4 & $\mathrm{H}_{3} \mathrm{PO}_{4}$ & -3.5 & $2.71 \mathrm{mM}$ & Lys1630, Arg1655, Glu1570 & Glu1570, Lys1630, Arg1655 \\
\hline 5 & $\mathrm{NaCl}$ & -0.99 & $186.80 \mathrm{mM}$ & Trp1585, Met1596, Ile1643, Thr1648 & - \\
\hline 6 & $\mathrm{KCl}$ & -0.83 & $247.63 \mathrm{~m}$ & Trp1585, Met1596, Leu1601, Ile1643, Thr1648 & - \\
\hline 7 & $\mathrm{Ca}\left(\mathrm{NO}_{3}\right)_{2}$ & -4.39 & $601.5 \mathrm{uM}$ & Asn1638, Phe1639, Gly1641, Ser1684, Lys1688, Tyr1697 & $\begin{array}{l}\text { Asn1638, Gly1641, } \\
\text { Lys1688, Ser1689 }\end{array}$ \\
\hline 8 & TSP & -4.46 & $537.44 \mathrm{uM}$ & Phe1639, Gln1640, Arg1642, His1644, Asp1647, Lys1668, Asp1685 & Asp1547, Lys1668, Asp168 \\
\hline 9 & $\mathrm{KNO}_{3}$ & -3.2 & $4.53 \mathrm{mM}$ & Tyr1594, Trp1675, Lys1676, Phe1677, Glu1873 & Asn1681 \\
\hline 10 & $\left(\mathrm{NH}_{4}\right)_{2} \mathrm{SO}_{4}$ & -3.06 & $5.76 \mathrm{mM}$ & His1644, Asn 1671, Asn1681, Glu1683, Ser1684 & Gln349, Asp392, Asp394 \\
\hline 11 & $\mathrm{NaNO}_{3}$ & -3.77 & $1.74 \mathrm{mM}$ & Lys1688, Ser1689, Ser1694, Tyr1697 & Lys1688 \\
\hline 12 & MAP & -2.82 & $8.59 \mathrm{mM}$ & Phe1687, Lys1688 & Phe1687, Lys1688 \\
\hline 13 & DAP & -2.9 & $7.53 \mathrm{mM}$ & Asn1629, Lys1630, Ser1633 & Asn1629, Lys1630 \\
\hline 14 & $\mathrm{MCP}$ & -3.97 & $1.29 \mathrm{mM}$ & Tyr1595, Phe1639, Gln1640, Arg 1642, His1644 & Asp1685 \\
\hline
\end{tabular}

SSP: Single Sugar Phosphate; TSP: Triple Sugar Phosphate; MAP: monoammonium phosphate;

DAP: Diammonium phosphate; MCP: Monocalcium phospate 


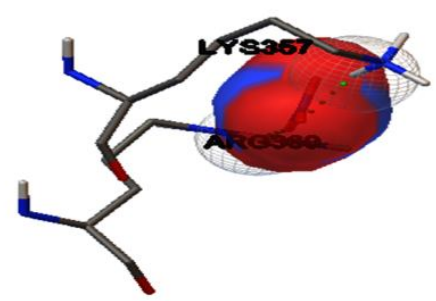

Ammonium nitrate

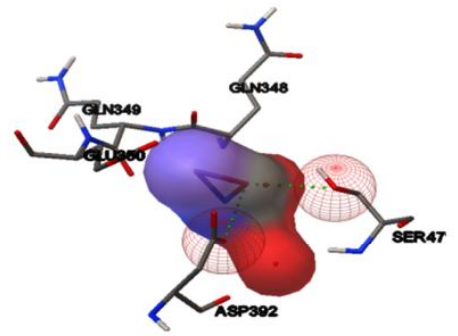

Potassium nitrate

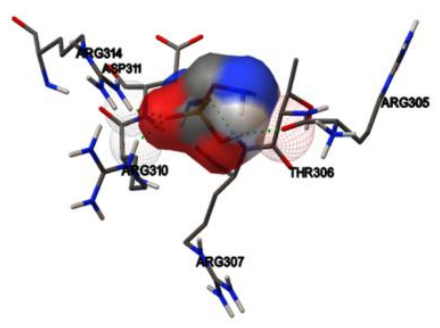

Diammonium phosphate

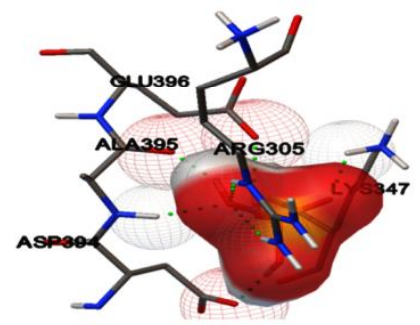

Phosphoric acid

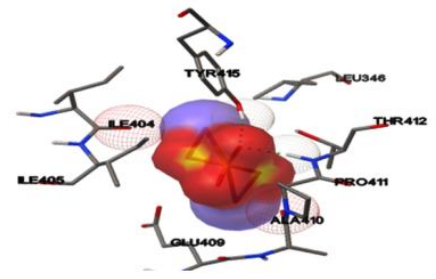

Potassium sulphate

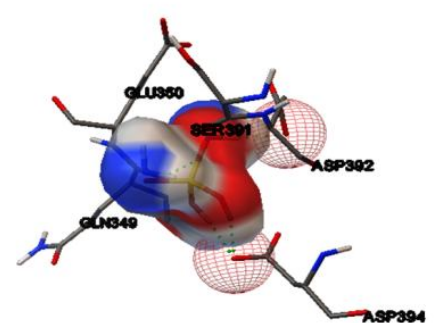

Ammonium sulphate

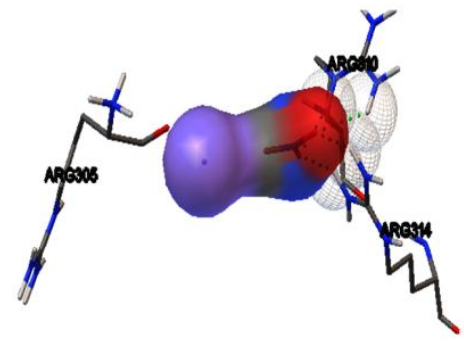

Sodium nitrate

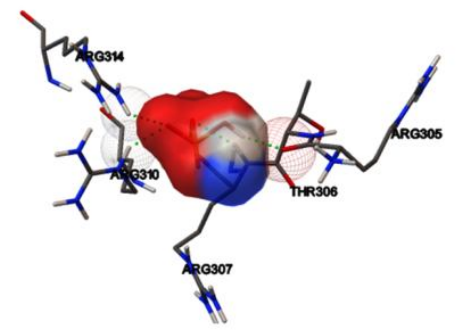

Monoammoniumphosphate

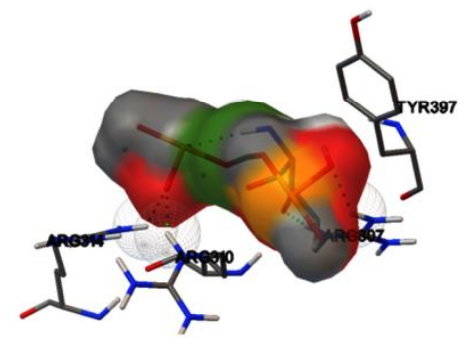

Singlesuper phosphate

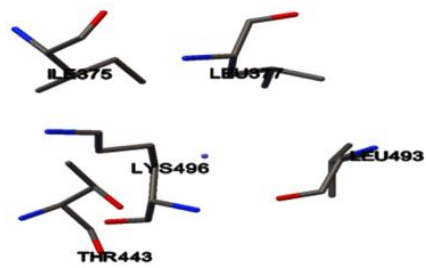

Potassium chloride

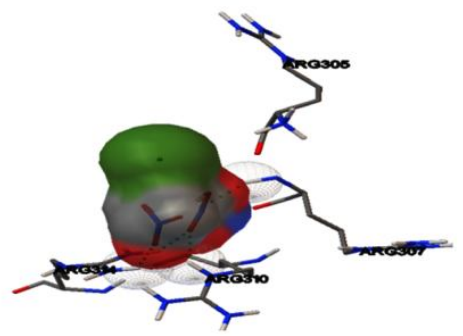

Calcium ammonium nitrate

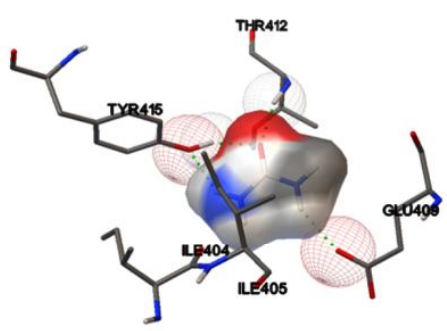

Urea

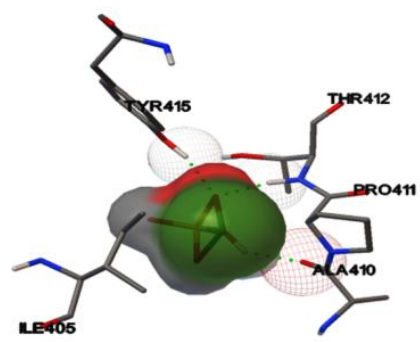

Monocalciumphosphate

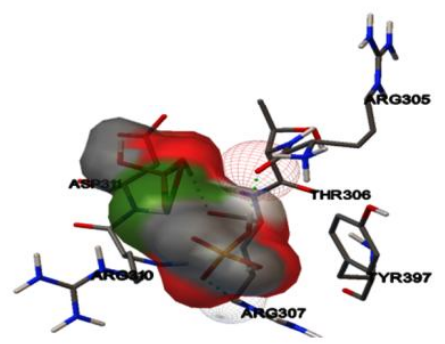

Triplesuper phosphate

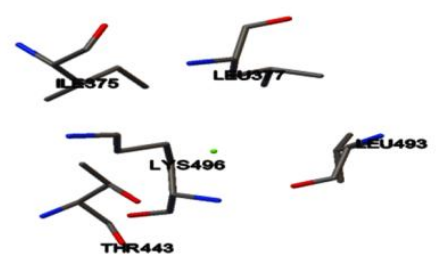

Sodium chloride

Fig. 2a: Docking results showing interaction of VGKC(AtKat1) with different fertilizers 


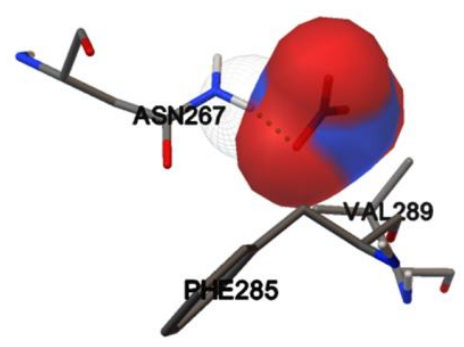

Ammonium nitrate

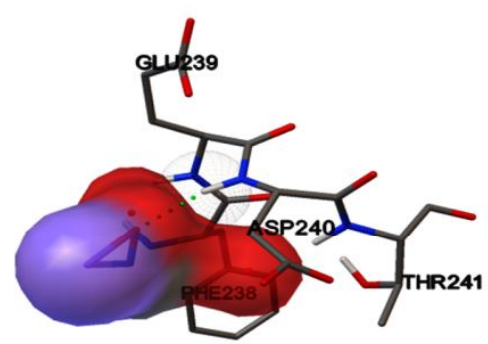

Potassium nitrate

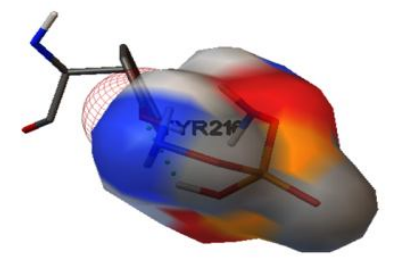

Diammonium phosphate

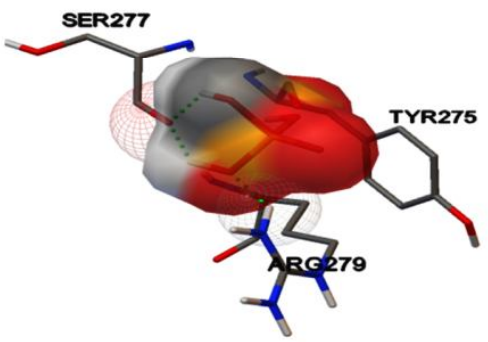

Phosphoric acid

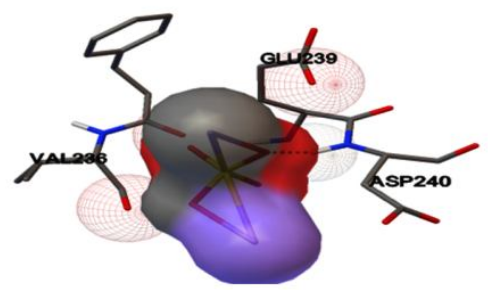

Potassium sulphate

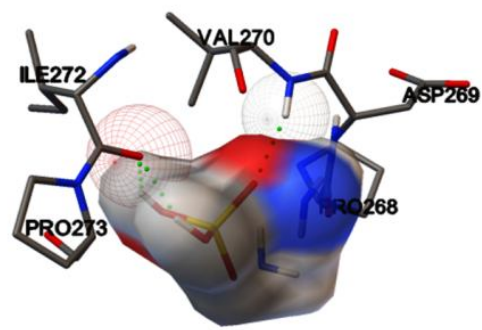

Ammonium suphate

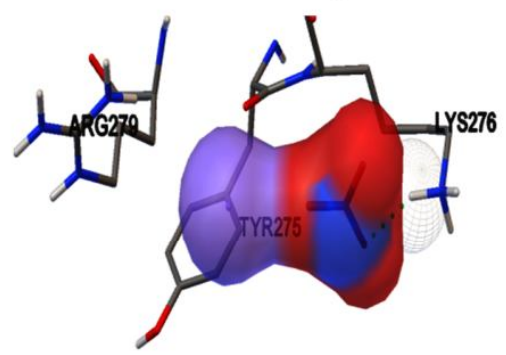

Sodium nitrate

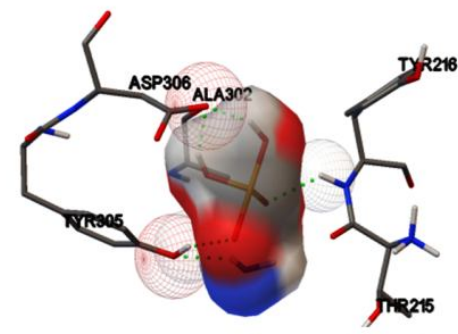

Mono-ammonium phosphate

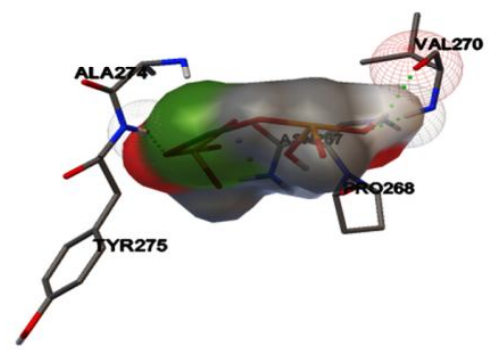

Single super phosphate

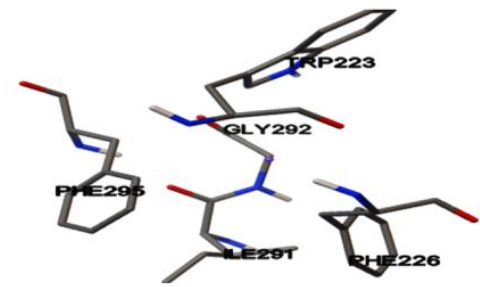

Potassium chloride

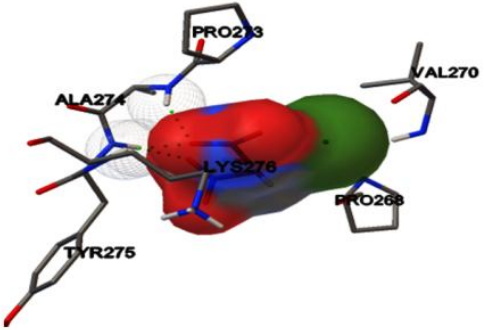

Calcium ammonium nitrate

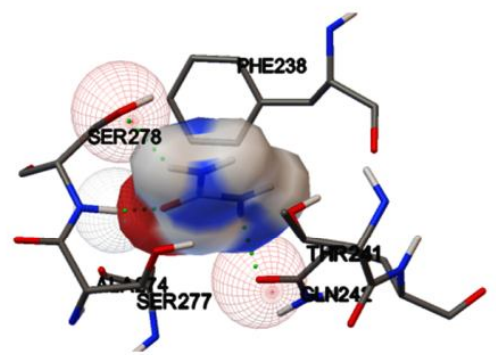

Urea

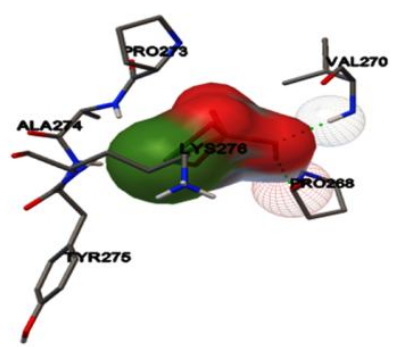

Mono-calcium phosphate

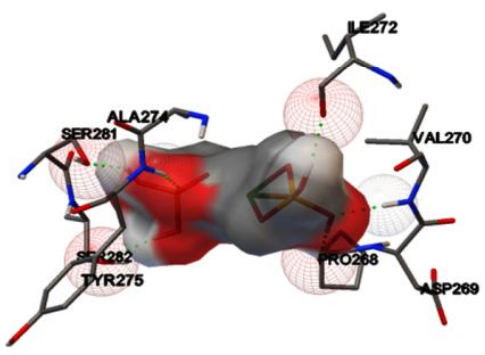

Triple super phosphate

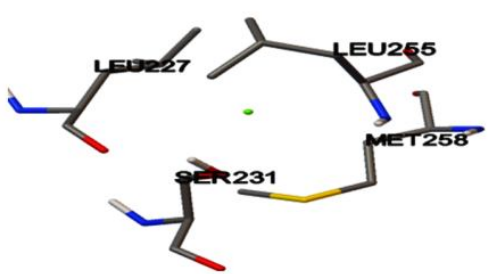

Sodium chloride

Fig. 2b: Docking results showing interaction of $\mathrm{VGCC}(\mathrm{AtTpc} 1)$ with different fertilizers 


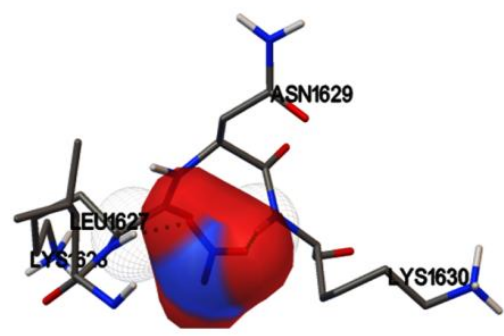

Ammonium nitrate

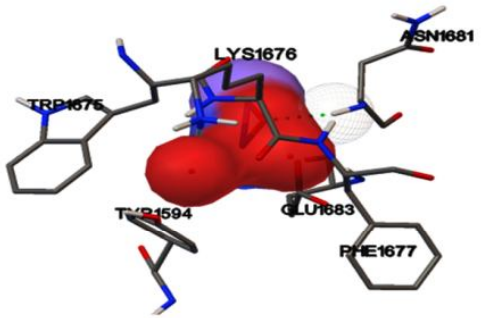

Potassium nitrate

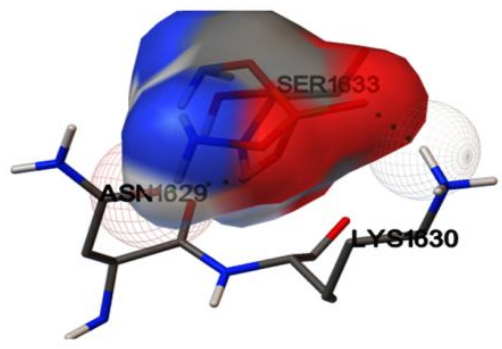

Diammonium phosphate

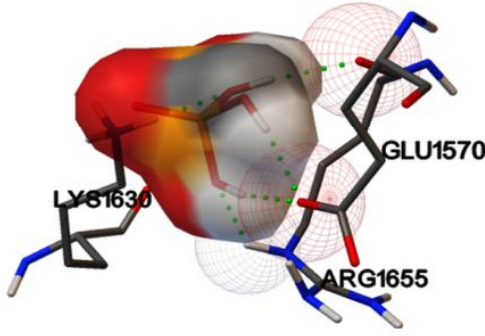

Phosphoric acid

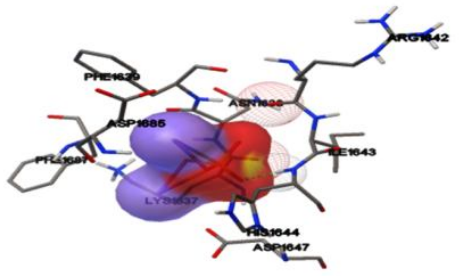

Potassium sulphate

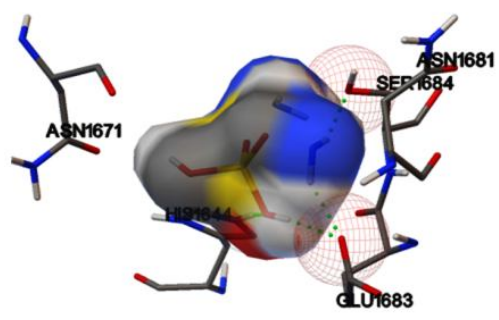

Ammonium sulphate

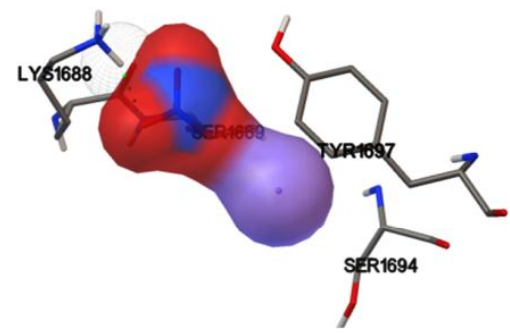

Sodium nitrate

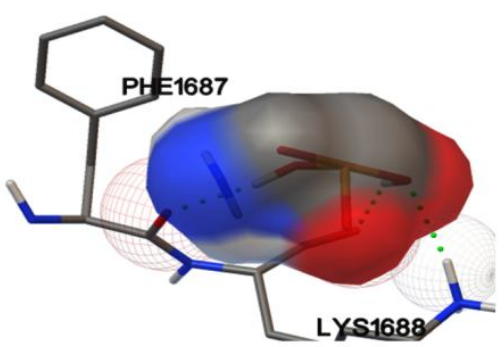

Mono-ammonium phosphate

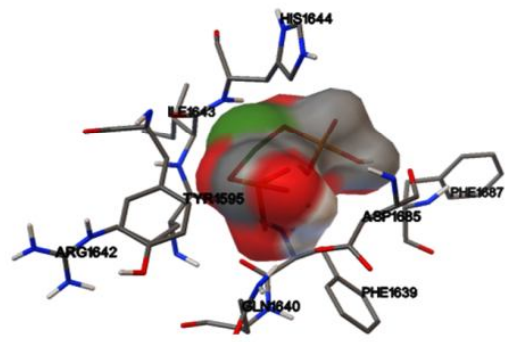

Single super phosphate

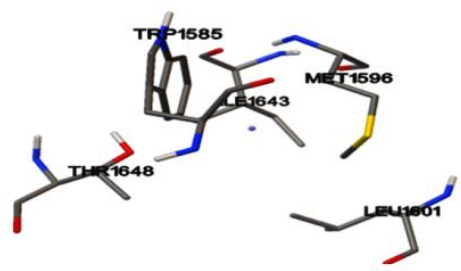

Potassium chloride

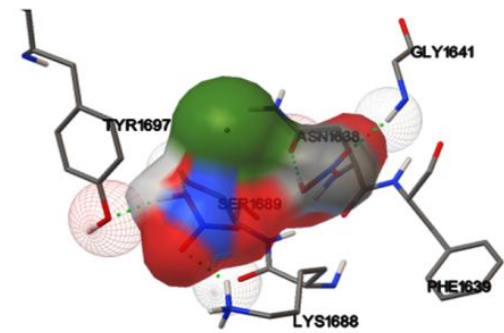

Calcium ammonium nitrate

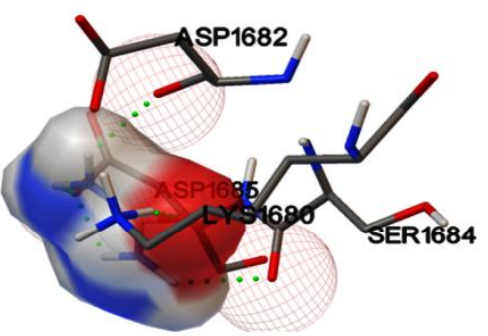

Urea

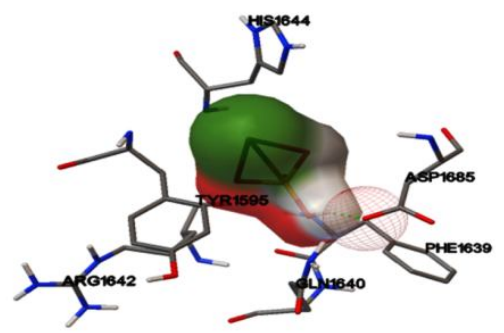

Mono-calcium phosphate

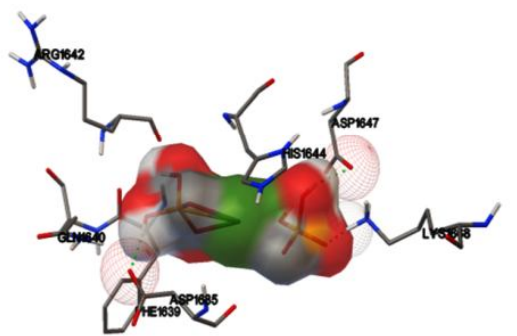

Triple super phosphate

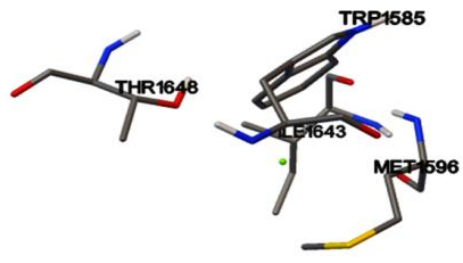

Sodium chloride

Fig. 2c: Docking results showing interaction of VGSC(Mpccmp1545) with different fertilizers 
Table. 5: Docking analysis of VGKC of A. thaliana (AtKat1) against selected soil salts and fertilizers at different temperature.

\begin{tabular}{|c|c|c|c|c|c|c|c|c|c|}
\hline \multirow{2}{*}{ S.No. } & \multirow{2}{*}{ Salt name } & \multicolumn{8}{|c|}{ Binding Energy (kCal/mol) } \\
\hline & & $10^{\circ} \mathrm{C}$ & $15^{0} \mathrm{C}$ & $25^{0} \mathrm{C}$ & $30^{\circ} \mathrm{C}$ & $35^{\circ} \mathrm{C}$ & $40^{\circ} \mathrm{C}$ & $45^{\circ} \mathrm{C}$ & $50^{\circ} \mathrm{C}$ \\
\hline 1 & $\mathrm{NH}_{4} \mathrm{NO}_{3}$ & -4.26 & -4.29 & -4.24 & -4.25 & -4.3 & -4.3 & -4.3 & -4.26 \\
\hline 2 & $\mathrm{H}_{3} \mathrm{PO}_{4}$ & -3.16 & -3.29 & -3.53 & -3.1 & -2.78 & -2.89 & -2.49 & -4.26 \\
\hline 3 & $\mathrm{NaCl}$ & -1.01 & -1.01 & -1.01 & -1.01 & -1.01 & -1.01 & -1.01 & -1.01 \\
\hline 4 & Triple super phosphate & -2.52 & -2.86 & -2.97 & -2.69 & -2.69 & -2.92 & -2.94 & -2.69 \\
\hline 5 & $\left(\mathrm{NH}_{4}\right)_{2} \mathrm{SO}_{4}$ & -2.26 & -2.23 & -1.91 & -2.17 & -2.24 & -2.19 & -2.42 & -2.22 \\
\hline
\end{tabular}

Table. 6: Docking analysis of VGCC of A. thaliana (AtTpc1) against selected soil salts and fertilizers at different temperature.

\begin{tabular}{|c|c|c|c|c|c|c|c|c|c|}
\hline \multirow[b]{2}{*}{ S.No. } & \multirow[b]{2}{*}{ Salt name } & \multicolumn{8}{|c|}{ Binding Energy (kCal/mol) } \\
\hline & & $10^{0} \mathrm{C}$ & $15^{0} \mathrm{C}$ & $25^{0} \mathrm{C}$ & $30^{0} \mathrm{C}$ & $35^{0} \mathrm{C}$ & $40^{\circ} \mathrm{C}$ & $45^{\circ} \mathrm{C}$ & $50^{0} \mathrm{C}$ \\
\hline 1 & $\mathrm{NH}_{4} \mathrm{NO}_{3}$ & -3.23 & -3.17 & -3.22 & -3.25 & -3.25 & -3.22 & -3.22 & -3.23 \\
\hline 2 & $\mathrm{H}_{3} \mathrm{PO}_{4}$ & -2.2 & -2.02 & -2.18 & -2.1 & -2.03 & -2.34 & -2.15 & -3.22 \\
\hline 3 & $\mathrm{NaCl}$ & -1.05 & -1.05 & -1.05 & -1.05 & -1.05 & -1.05 & -1.05 & -1.05 \\
\hline 4 & Triple super phosphate & -2.43 & -2.17 & -2.46 & 2.46 & -2.13 & -2.35 & -2.45 & -2.44 \\
\hline 5 & $\left(\mathrm{NH}_{4}\right)_{2} \mathrm{SO}_{4}$ & -2.29 & -2.01 & -1.54 & -1.98 & -1.81 & -2.04 & -1.88 & -2.00 \\
\hline
\end{tabular}

Table. 7: Docking analysis of VGSC of $A$. thaliana (Mpccmp1545) against selected soil salts and fertilizers at different temperature.

\begin{tabular}{|c|c|c|c|c|c|c|c|c|c|}
\hline \multirow{2}{*}{ S.No. } & \multirow{2}{*}{ Salt name } & \multicolumn{8}{|c|}{ Binding Energy (kCal/mol) } \\
\hline & & $10^{0} \mathrm{C}$ & $15^{0} \mathrm{C}$ & $25^{0} \mathrm{C}$ & $30^{0} \mathrm{C}$ & $35^{0} \mathrm{C}$ & $40^{\circ} \mathrm{C}$ & $45^{0} \mathrm{C}$ & $50^{0} \mathrm{C}$ \\
\hline 1 & $\mathrm{NH}_{4} \mathrm{NO}_{3}$ & -1.75 & -1.73 & -1.74 & -1.75 & -1.74 & -1.75 & -1.76 & -1.75 \\
\hline 2 & $\mathrm{H}_{3} \mathrm{PO}_{4}$ & -2.25 & -2.25 & -2.21 & -2.19 & -2.15 & 2.51 & -2.35 & -3.75 \\
\hline 3 & $\mathrm{NaCl}$ & -1 & -1 & -1 & -1 & -1 & -1 & -1 & -1 \\
\hline 4 & Triple super phosphate & -3.49 & -2.8 & -2.5 & -2.72 & -2.94 & -2.7 & -2.87 & -2.22 \\
\hline 5 & $\left(\mathrm{NH}_{4}\right)_{2} \mathrm{SO}_{4}$ & -1.93 & -1.71 & -1.42 & -1.57 & -1.73 & -1.49 & -2.17 & -1.79 \\
\hline
\end{tabular}

\section{CONCLUSION}

The present study is novel in itself being probably the first comprehensive study about role of VGICs in plant system with special reference to abiotic stress (salinity and temperature) response. The study has provided a good platform for further investigation to establish the role of VGICs in plant stress response and correlated to other living systems like animals, fungi, etc.

\section{ACKNOWLEDGEMENT}

The authors acknowledge the Bioinformatics Infrastructure Facility (Supported by Dept. of Biotechnology, Govt. of India), M.D. University, Rohtak, for extending computational facility for the present work.

\section{REFERENCES}

1. Armstrong CM, Hille B. Voltage-Gated Ion Channels and Electrical Excitability. Neuron. 1998; 20: 371-380.

2. Noda M, Shimuzu S, Tanabe T, Takai T, KayanoT, Ikeda T, Takahashi H, Nakayama H, Kanaoka Y, Minamino N, Kengawa K, Matsuo H, Raftery M, Hirose T, Inayama S, Hayashida H, Miyata T, Numa S. Primary structure of Electrophorus electricus sodium channel deduced from cDNA sequence. Nature.1984; 312: 121-127.

3. Noda M, Ikeda T, Kayano T,Suzuki H, Takeshima H, Kurasaki, Takahashi H, Numa S. Existence of distinct sodium channel messenger RNAs in rat brain. Nature. 1986; 320: 188- 192.

4. Tempel TM, Papazian DM, Schwarz TL, Jan YN, Jan LY. Sequence of a probable potassium channel component encoded at Shaker locus in drosophila. Science. 1987; 237: 770-775.

5. Amtmann A, Armengaud P, Volkov V. Potassium nutrition and salt stress. In: Blatt MR (ed) Membrane transport in plants. Blackwell Oxford; 2004, p. 293-339.
6. Serrano R, Mulet JM, Rios G, Marquez JA, de Larrinoa IF, Leube MP, Mendizabal I, Pascual-Ahuir A, Proft M, Ros R, Montesinos C. A glimpse of the mechanisms of ion homeostasis during salt stress. J Exp Bot. 1999; 50:1023-1036.

7. Wyn Jones RJ, Pollard A. Proteins, enzymes and inorganic ions. Encyclopedia of Plant Physiology, Springer. 1983; 126: 528-562.

8. Hepler PK, Wayne RO. Calcium and plant development. Annu. Rev. Plant Physiol. 1985; 36: 397-439.

9. Leonard RT, Hepler PK. Eds. Calcium in Plant Growth and Development. Rockville, MD: American Society of Plant Physiologists; 1990.

10. Thuleau P, Ward JM, Ranjeva R, Schroeder JI. Voltage dependent calcium-permeable channels in the plasma membrane of a higher plant cell. EMBO J. 1994; 13: 2970-2975.

11. Huang JW, Grunes DL, Kochian LV. Voltage dependent Ca2+ influx into right-side-out plasma membrane vesicles from wheat roots: Characterization of a putative $\mathrm{Ca}^{2+}$ channel. Proc. Natl. Acad. Sci. USA. 1994; 91: 3473-3477.

12. Marshall J, Corzo A, Lelgh RA, Sanders D. Membrane potential dependent calcium transport in right-side-out plasma membrane vesicles from Zea mays L. roots. Plant J. 1994; 5: 683694.

13. Pińeros M, Tester M. Characterization of a voltage dependent $\mathrm{Ca}^{2+}$-selective channel from wheat roots. Planta. 1995; 195: 478488.

14. Ward JM, Maser P, Schroeder JI. Plant Ion Channels: Gene Families, Physiology and Functional Genomics Analyses. Annual review of physiology. 2009; 71: 59-82.

15. McAinsh MR, Brownlee C, Hetherington AM. Calcium ions as second messengers in guard cell signal transduction. Physiologia Plantarum. 1997; 100: 16-29.

16. Grabov A, Blatt MR. Membrane voltage initiates $\mathrm{Ca}^{2+}$ waves and potentiates $\mathrm{Ca}^{2+}$ increases with abscisic acid in stomatal guard cells. Proceedings of the National Academy of Sciences, USA. 1998; 95: 4778-4783.

17. White PJ. Calcium channels in the plasma membrane of root cells. Annals of Botany. 1998; 81: 173-183. 
18. White PJ, Biskup B, Elzenga JTM, Homann U, Thiel G, Wissing F, Maathuis FJM. Advanced patch-clamp techniques and singlechannel analysis. Journal of Experimental Botany. 1999; 50:1037-1054.

19. Ji-Ping Gao, Dai-Yin Chao, Hong-Xuan Lin. Understanding Abiotic Stress Tolerance Mechanisms: Recent Studies on Stress Response in Rice. Journal of Integrative Plant Biology. 2007; 49(6): 742-750.

20. NCBI: https://www.ncbi.nlm.nih/gov

21. SWISS MODEL: https://www.swissmodel.expasy.org

22. Hekkelman ML, Beek TA, Pettifer SR, Thorne D, Attwood, Virend G. WIWS: a protein structure bioinformatics Web service collection. Nuc. Ac. Res. 2010; 38: W719-723.

23. UCSF-Chimera: https://www.cgl.ucsf.edu/chimera/
24. Chemsketch: www.acdlabs.com/resources/freeware/chemsketch/

25. Open Babel: https://www. openbabel.org/

26. Morris GM, Huey R, Lindstrom W, Sanner MF, Belew RK, Goodsell DS, Olson AJ. Autodock4 and AutoDockTools4: automated docking with selective receptor flexibility. J. Computational Chemistry. 2009; 16: 2785-91.

\section{How to cite this article:}

Ravi Ranjan Kr. Niraj, Nikita Singh, Sween, Ajit Kumar. Homology Modelling and Molecular Docking Study of Voltage Gated Ion Channels for their Role in Plant Abiotic Stress. J App Biol Biotech, 2014; 2 (04): 007-015. 